\title{
Augmented phytotoxic effect of nanoencapsulated Ophiobolin A
}

M. Vurro ${ }^{1 *}$, H. E.Townley², Rachel Morrison, Angela Boari ${ }^{1}$, Marco Masi, A. Evidente ${ }^{3}$ ${ }^{1}$ Institute of Sciences of Food Production, National Research Council, via Amendola 122/O, 70125 Bari, Italy

2Department of Engineering Science, Oxford University, Parks Road, Oxford, UK ${ }^{3}$ Department of Chemical Sciences, University of Naples "Federico II", Complesso Universitario Monte S. Angelo, Via Cintia 4, 80126 Napoli, Italy

Corresponding author: Maurizio Vurro. e-mail: maurizio.vurro@ispa.cnr.it 
Abstract

Keywords: Ophiobolin A; mesoporous silica nanoparticles; herbicidal activity 


\section{Introduction}

Despite the potential of natural metabolites to be used as safe and environmentally friendly agrochemicals (Copping and Duke, 2007; Cantrell et al., 2012), some of their characteristics (e.g. excessive biodegradability, difficulty to reach or penetrate the target, slow action, need of high rates of application) often represent limiting factors for their practical application.

Nanomaterials may present a solution to overcome some of these limiting factors, protecting the compounds from environmental conditions, increasing stability, modulating release kinetics, and targeting application to weeds, diseases or pests (Vurro et al., 2019).

The discovery of highly ordered mesoporous silica materials (Mobil Corporation in 1992) led to a variety of important applications. Mesoporous silica nanoparticles (MSNPs) are synthetic particles possessing well defined and tunable pore sizes $(2-50 \mathrm{~nm})$, large pore volumes, high surface areas with easily-modifiable surface properties, chemical stability, resistance to microbial attack, tailorable nanostructures, biocompatibility, and aqueous degradability. Moreover, MSNPs protect loaded active ingredients (Als) against enzymatic degradation as no swelling or changes in porosity occur in response to external stimuli, such as $\mathrm{pH}$ and temperature (ref).

MSNPs possess external and internal surfaces that can be selectively functionalized with multiple organic and inorganic groups. Due to their characteristics, MSNPs have been particularly embraced by the pharmaceutical medicine sector for drug encapsulation and controlled release of drugs and antigens (Huang et al, 2020 a, b) into mammalian living cells, or anti-cancer compounds which can be delivered directly to the site of a tumour, reducing systemic side effects and enabling a higher dose to be reached in the cancerous tissue (Huang et al., 2017).

Much of what has been learned from nanomedicine can be applied to generate nanoformulations for use in agriculture, and MSNPs could be particularly suitable as delivery carriers for bioactive natural metabolites, by virtue of their porous structure, chemical stability, biocompatibility (Slowing et al., 2008), tuneable pore size and porosity (Trewyn et al., 2007), simple and low-cost synthesis (Kwon et al., 2013), and potential scale-up for industrial use. Additionally, silica is biologically inert and has the ability to decompose into relatively harmless silicic acid byproducts (Diaconu et al., 2010), making it a useful material for biocide delivery applications in agriculture. An additional advantage of MSNPs is that they can be capped to further protect the encapsulated metabolites against degradation or evaporation so enabling a controlled and prolonged release of the agrochemicals. Recently, MSNPs were used to encapsulate essential oils, and proved to provide protection and prevent volatilization of the oils, whilst improving their stability, long-term effects, and immiscibility in aqueous solutions (Bravo et al, 2018 a,b).

However, unlike mammalian cells, plant cells possess walls acting as a barrier for easy entry of any external agent including nanoparticles into plant cells. The sieving properties are determined by the pore diameter of the cell wall which ranges from 5 to $20 \mathrm{~nm}$. Hence, only nanoparticles or nanoparticle aggregates with diameters less than the pore diameter of the cell wall could easily pass 
through and reach the plasma membrane. When nanoparticles are applied on leaf surfaces, they enter through the stomatal openings or through the bases of trichomes and are then translocated to various tissues. Conversely, larger nanoparticles can take advantage of the presence of small lesions on the leaf surface that could favour penetration and uptake. This could be particularly advantageous for nanoparticles carrying metabolites which have herbicidal properties.

Despite these promising results, little has been studied with regards to the possibility to use MSNPs to encapsulate natural products having herbicidal properties, in order to improve their efficacy and modulate the release in to the target.

Ophiobolin A () (figure 1) is a secondary metabolite produced by some phytopathogenic fungal species responsible for severe diseases, e.g. of rice, maize, and sorghum, on which they produce brown spot leaf lesions. This sesterterpene-type compound is one of a group of over 50 ophiobolin analogues, which all feature a unique tricyclic 5-8-5 ring system named as A-, B- and C-ring, respectively (Au et al. 2000; Masi et al., 2019). Recently, ophiobolin was purified from D. gigantean and two new ophiobolins isolated, named ophiobolin A and $B$, which have been characterized for both phytotoxic activities and anticancer properties (Zatout et al., 2020).

Ophiobolins cause numerous detrimental effects when applied to plants. The growth of roots and coleoptiles of rice seedlings have been shown to be inhibited at very low concentrations of ophiobolin. The activity of phloroglucinoloxidase, a marker enzyme for fungal infection, increased in rice leaves when treated with ophioboim. Furthermore, some ophiobolins could reduce the growth of roots and coleoptiles of wheat seedlings, reduce seed germination, change cell membrane permeability, stimulate leakage of ß-cyanin, electrolytes and glucose from roots, decrease photosynthetic $\mathrm{CO}_{2}$-fixation, cause respiratory changes and enhance stomatal opening (Au et al. 2000). Ophiobolins are also considered to play a role in disease development since the characteristic brown spot lesions on leaves could be induced by the application of droplets of the toxins to the plant leaves (Evidente et al., 2006a; Evidente et al. 2006b).

Besides the research on ophiobolins historically-focused on plants (Au et al. 2000), more recently they have shown a broad spectrum of inhibitory activities in vitro on various cancer cell lines (Bury et al., 2013a; Bury et al., 2013b). Chemoembolization particles have been designed and synthesized for the delivery of ophiobolin OA directly to the tumour location (Morrison et al., 2017). Analogues of Ophiobolin A were synthesized to aid in the determination of the moe of action. . Reaction with reaction primary amines suggested that pyrrolylation of lysine residues on its intracellular target protein(s) and/or phosphatidylethanolamine lipid is responsible for its biological effects (Masi et al. 2019).

Herein we investigated whether the phytotoxic activities of Ophiobolin A against weed species could be improved by nanoencapsulation. Given the rapid natural degradation of the compound (Morrison et al. ) it was hoped that nanoencapsulation would prolong the phytotoxic effects leading 
to better weed control. This article presents an assessment of the pure compound on 11 commonly found weed species and compared the activity with nanoparticle encapsulated Ophiobolin A. 


\section{Material and Methods}

\subsection{Production and purification of pure Ophiobolin A}

OA was produced by growing Drecslera gigantea on a mineral defined medium as previously reported (Evidente et al., 2006). The fungal culture filtrates were extracted and the organic extract fractionated on column chromatography as previously described, obtaining opiobolin $A$ in the form of white crystals (Evidente et al., 2006)

\subsection{Nano-encapsulation}

MSNPs were prepared according to the method of Hom et al., (2010). Briefly, $100 \mathrm{mg}$ Cetyl

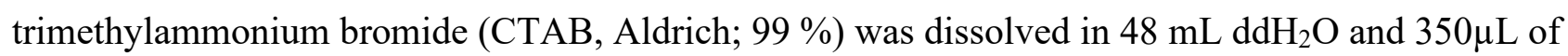
$2 \mathrm{M} \mathrm{NaOH}$ and vigorously stirred in a round-bottom flask at $80^{\circ} \mathrm{C}$. After the temperature was stabilized, $500 \mu \mathrm{L}$ Tetraethyl orthosilicate (TEOS, Aldrich) was added. After a further two hours incubation, the nanoparticles were collected by centrifugation and washed twice with methanol. The CTAB surfactant was removed by overnight reflux in acidic methanol $(20 \mathrm{~mL}$ methanol, $1 \mathrm{~mL} 37$ $\%$ hydrochloric acid) at $80{ }^{\circ} \mathrm{C}$.

The MSNPs (40 mg) were loaded with $8 \mathrm{~mL}$ of $0.5 \mathrm{mg} / \mathrm{mL}$ ophiobolin A in dichloromethane. Samples were mixed on a magnetic stirrer at $500 \mathrm{rpm}$ for 3 days at room temperature. The nanoparticles were then pelleted at $12000 \mathrm{rpm}$ for 5 minutes and the supernatant removed. The loaded MSNP@Ophiobolin A were then freeze dried.

Hom, C., J. Lu, M. Liong, H. Luo, Z. Li, et al. (2010) Mesoporous Silica Nanoparticles Facilitate Delivery of siRNA to Shutdown Signaling Pathways in Mammalian Cells. Small, 6(11): p. 11851190

\subsection{Bioassay}

\subsubsection{Plant material}

The plant species to be used in the assays were chosen both among weedy dicots and monocots, all belonging to very important genera. They were also chosen according to represent different leaf characteristics, e.g. thick or thin epidermis, smooth or hairy leaf surface, deep waxy etc. Plants were harvested from populations growing in naturally infested fields in Bari (Southern Italy) countryside. Uniform leaf disks were cut from fully-expanded and healthy leaves using a cork borer ( $\varnothing 8 \mathrm{~mm}$ ). Leaf disks were then immediately placed in polycarbonate boxes on wet filter paper on suitable trays and used as soon as possible for the treatments. 
Those plant species investigated are listed in table 1. The first experiment used plants numbered 19, whereas the second experiment used all 11 plants listed.

Table 1. List of plant species whose leaves where used in the 2 experiments

\begin{tabular}{|c|c|c|c|c|}
\hline \# & Specific name & Trivial name & Family & Leaf features \\
\hline 1 & Cirsium arvense (L.) Scop. & Canada thistle & Asteraceae & $\begin{array}{l}\text { very spiny, lobed, hairy } \\
\text { beneath }\end{array}$ \\
\hline 2 & Convolvulus arvensis $\mathrm{L}$. & Field bindweed & Convolvulaceae & $\begin{array}{l}\text { Glabrous with scattered } \\
\text { crisped hairs }\end{array}$ \\
\hline 3 & $\begin{array}{l}\text { Helminthotheca (Picris) } \\
\text { echioides (L.) Holub }\end{array}$ & bristly oxtongue & Asteraceae & $\begin{array}{l}\text { Stiff hairs are sometimes } \\
\text { hooked at the apex }\end{array}$ \\
\hline 4 & Brassica nigra L. & Black mustard & Brassicacee & Partly bristly hairy \\
\hline 5 & Urtica urens L. & Annual nettle & Urticaceae & $\begin{array}{l}\text { Both sides with scattered } \\
\text { stinging hairs }\end{array}$ \\
\hline 6 & Papaver rhoeas L. & Red poppy & Papaveraceae & Hairy \\
\hline 7 & $\begin{array}{l}\text { Erodium malacoides (L.) } \\
\text { L'Hér. }\end{array}$ & $\begin{array}{l}\text { Mediterranean stork's } \\
\text { bill }\end{array}$ & Geraniaceae & $\begin{array}{l}\text { Covered with fine } \\
\text { pubescence }\end{array}$ \\
\hline 8 & Avena fatua $\mathrm{L}$. & Common wild oat & Poaceae & $\begin{array}{l}\text { Hairless lamina with } \\
\text { parallel veins and ciliated } \\
\text { edges }\end{array}$ \\
\hline 9 & Galium aparine L. & Cleavers & Rubiaceae & $\begin{array}{l}\text { Stiff hairs on the margins } \\
\text { and a single central vein }\end{array}$ \\
\hline 10 & $\begin{array}{l}\text { Dittrichia viscosa (L.) } \\
\text { Greuter }\end{array}$ & Sticky fleabane & Asteraceae & Elongated and sticky \\
\hline 11 & Lantana camara L. & Big sage - Tickberry & Verbenaceae & $\begin{array}{l}\text { Strigose upper surface, } \\
\text { deeply vined }\end{array}$ \\
\hline
\end{tabular}

\subsubsection{Treatments}

Each leaf disk received $20 \mu$ of the solutions prepared as described above. Four replicates were prepared for each treatment and for each plant species. The treated leaf discs were placed in sealed transparent boxes and incubated in a plant growth chamber at $\sim 25^{\circ} \mathrm{C}$ under continuous light. Symptoms of phytotoxicity were evaluated 2 days after treatment (DAT), when the effects were already clearly distinguishable and the remaining parts of the disks were still mostly healthy. Disks were observed up to 5 DAT to confirm day 2 results, or to observe the symptom evolution.

Depending on the treatment (see above), the leaf surface was left intact or injured by using an insulinsyringe needle, thus provoking small and superficial wounds (scratch), immediately before droplet application; depending on the bioassay, both procedures (intact or scratched) were used for both the upper or the lower leaf sides, as mentioned above.

Considering the amount of OA loaded into the nanoparticles (a rate of 1 to 8.7 in weight), a suitable concentration containing an amount of OA sufficient to be active in the assay, contained by a manageable amount of nanoparticles was determined from preliminary dosing experiments. Before leaf application the particles were resuspended in DMSO (2 or $4 \%$ of the final concentration). Thus, the nano-formulated OA solution tested had a final concentration of $0.2 \mu \mathrm{g} \mathrm{OA} / \mu$ l solution and 1.74 $\mu \mathrm{g} / \mu \mathrm{l}$ nanoparticles (MNP). For the comparative studies, solutions containing OA alone $(0.2 \mu \mathrm{g} / \mu \mathrm{l})$, 
nanoparticles alone $(1.71 \mu \mathrm{g} / \mu \mathrm{l})$, or nanoparticles mixed exogenously to OA were prepared. All the solutions were prepared a few minutes before the use, to prevent early toxin release.

An empiric and comparative scale of toxicity was used, where $0=$ no symptoms; $-/+=$ traces of necrosis; + = small necrosis clearly visible; $++=$ quite large and evident necrosis, wider than the wounded zone; +++ = even wider necrosis.

\subsubsection{First Treatment}

In this first assay, the leaf discs were subjected to 9 different treatments, as shown in the table 2. For each treatment, four disks of the plants designated numbers 1 to 9 (table 1 ) were used. Thus, the experimental scheme was the following:

Table 2. Solutions applied to leaf disks in Experiment 1

\begin{tabular}{|l|l|l|}
\hline$\#$ & Treatment & Type of application \\
\hline 1 & MNP@OA & Encapsulated ophiobolin \\
\hline 2 & MNP@OA + Scratch & Encapsulated ophiobolin applied to wounded disks \\
\hline 3 & OA & Ophiobolin A alone \\
\hline 4 & OA + Scratch & Ophiobolin applied to wounded disks \\
\hline 5 & MNP & Nanoparticles alone \\
\hline 6 & MNP + Scratch & Nanoparticles applied to wounded disks \\
\hline 7 & DMSO (2\%) & Control solution with 2\% DMSO (dimethylsulfoxide) \\
\hline 8 & DMSO + Scratch & Control solution with DMSO applied to wounded disks \\
\hline 9 & Water & Control - only water \\
\hline
\end{tabular}

\subsubsection{Second Treatment}

Considering the results of the first bioassay, in this second experiment all the leaf disks were wounded (scratched) as described in section 2.3.2. All the 11 plants species listed in Table 1 were used, preparing four disks per each plant. Thus, the experimental design shown in table 3 was used: Table 3. Experimental design of experiment 2

\begin{tabular}{|l|l|l|}
\hline$\#$ & Treatment & Type of treatment \\
\hline 1. & MNP@OA & Formulated ophiobolin \\
\hline 2. & OA & Ophiobolin alone \\
\hline 3. & MNP & Nanoparticles alone \\
\hline 4. & OA + MNP & Ophiobolin plus exogenously-added nanoparticles \\
\hline 5. & MNP@OA - abaxial & $\begin{array}{l}\text { Formulated ophiobolin applied to the abaxial leaf } \\
\text { side }\end{array}$ \\
\hline 6. & OA+MNP - abaxial & $\begin{array}{l}\text { Ophiobolin plus exogenously-added nanoparticles } \\
\text { applied to the abaxial leaf side }\end{array}$ \\
\hline 7. & MNP@OA - 1/5 rate & Formulated ophiobolin at a 1/5 rate compared to 1 \\
\hline
\end{tabular}


\begin{tabular}{|l|l|l|}
\hline 8. & Control DMSO $4 \%$ & Solution with solvent only \\
\hline
\end{tabular} 


\section{Results and discussion}

\section{Production and purification of Ophiobolin A}

Ophiobolin A was produced by fermentation on minimum mineral medium of $D$. gigantea and the culture filtrates was exhaustively extracted with ethyl acetate (EtOAc). The organic extract was then purified by silica gel column chromatography and the crude ophiobolin A crystallized by a mixture (1:5) EtOAc- $n$-hexane as white needles. Its physical and spectroscopic properties was compared to those already reported in the literature (Evidente et al., 2006a). The purity of OA > $98 \%$ was ascertained by $1 \mathrm{H}$ NMR, HPLC and ESI MS analyses.

\section{Nano-encapsulation}

The encapsulation of the ophiobolin A into the MSNPs was verified by liquid chromatography of the supernatant to assess the amount of drug which had not been incorporated, as discussed in Morrison et al., 2014.

\section{Bioassays - First treatment}

Table 4. Phytotoxic effects of different treatment with OA, MNP and wounds differently combined, on leaf disks of 9 plant species.

\begin{tabular}{|c|c|c|c|c|c|c|c|c|c|}
\hline \multirow{2}{*}{ Treatment } & 1 & 2 & 3 & 4 & 5 & 6 & 7 & 8 & 9 \\
& MNP@OA & MNP@OA +S & OA & OA+S & MNP & MNP+S & DMSO & DMSO+S & Water \\
\hline Plant & & & & & & & & & \\
\hline 1 & + & ++ & - & + & - & $-/+$ & - & - & - \\
\hline 2 & - & + & - & $-/+$ & - & - & - & - & - \\
\hline 3 & - & ++ & - & $-/+$ & - & - & - & - & - \\
\hline 4 & - & ++ & - & $-/+$ & - & - & - & - & - \\
\hline 5 & $-/+$ & + & - & $-/+$ & - & - & - & - & - \\
\hline 6 & - & ++ & - & $-/+$ & - & - & - & - & - \\
\hline 7 & - & + & - & $-/+$ & - & - & - & - & - \\
\hline 8 & - & ++ & $-/+$ & + & - & - & - & - & - \\
\hline 9 & + & + & - & $-/+$ & - & - & - & - & - \\
\hline
\end{tabular}

Where present (Table 4), the effects were clearly visible (figure ?) already 1 DAT. Necrosis was clearly delineated over the point of application, sometimes wider than the applied droplet. In general, the presence of scratches on the leaf surface proved to be necessary for symptoms to appear. Indeed, only plants 1 (C. arvense) and 9 (G. aparine) showed modest symptoms when the nanoencapsulated OA was applied without leaf injury. Conversely, when the formulation was applied over leaf wounds, necrotic areas were wide and evident in all the treated disks. At the concentration used, OA applied alone did not cause any toxicity. In previous work ophiobolin A had been used at higher concentrations, but limitations on the amount of OphA which could be loaded into 
nanoparticles and the subsequent amount of MSNPs which would need to be applied to the leaf surface was limiting. OA alone, applied after scratching the leaf surface proved to be modestly toxic to plants. Unloaded MSNPs were also applied after scratching and found to be non- toxic, although at the concentration used they formed a clear whitish deposit on the leaf surface. The protocol of the first experiment was chosen in order to: a) compare the effects of nanoencapsulated versus "pure" toxin; b) evaluate a possible advantage due to the presence of lesions (many toxins are very active only when they can penetrate or be absorbed by the plant tissues/leaf/root system); c) confirm the lack of toxicity of the nanoparticles alone, even when applied to wounded leaves.

Table 5. Phytotoxic effects of different treatment with OA, MSNPs and wounds differently combined, on leaf disks of 11 plant species.

\begin{tabular}{|c|c|c|c|c|c|c|c|c|}
\hline Treatment & $\begin{array}{c}1 \\
\text { MSNP@OA }\end{array}$ & $\begin{array}{c}2 \\
\text { OA }\end{array}$ & $\begin{array}{c}3 \\
\text { MSNP }\end{array}$ & $\begin{array}{c}4 \\
\text { OA + MNP }\end{array}$ & $\begin{array}{c}5 \\
\text { MSNP@OA } \\
- \text { abaxial }\end{array}$ & $\begin{array}{c}6 \\
\text { OA+ } \\
\text { MSNP - } \\
\text { abaxial }\end{array}$ & $\begin{array}{c}7 \\
\text { MSNP@OA } \\
-1 / 5\end{array}$ & $\begin{array}{c}8 \\
\text { Control }\end{array}$ \\
\hline Plant & & & & & & & & \\
\hline 1 & ++ & + & - & $-/+$ & + & - & + & - \\
\hline 2 & ++ & + & - & - & ++ & - & + & - \\
\hline 3 & + & - & - & - & ++ & - & + & - \\
\hline 4 & ++ & + & - & - & + & - & + & - \\
\hline 5 & ++ & + & - & - & ++ & - & + & - \\
\hline 6 & ++ & + & - & + & ++ & $-/+$ & + & - \\
\hline 7 & ++ & + & - & - & ++ & - & + & - \\
\hline 8 & + & - & - & - & ++ & - & + & - \\
\hline 9 & +++ & + & - & $-/+$ & ++ & $-/+$ & + & - \\
\hline 10 & ++ & + & - & $-/+$ & + & + & $-/+$ & - \\
\hline 11 & ++ & + & - & $-/+$ & ++ & $-/+$ & $-/+$ & - \\
\hline
\end{tabular}

Applied to wounded leaves, the nanoencapsulated toxin proved to be toxic to all plant species (table 5). Clearer and wider necrotic areas were visible on G. aparine, whereas smaller ones appeared on $H$. echioides and $A$. fatua. This result is in agreement with the leaf structure, as $A$. fatua is a monocot plant species in which the leaves have parallel veins and siliceous epidermis, and $H$. echioides has few stiff hairs. Thus, both leaf types are probably not very sensitive to wounds, and not very conducive to infiltration by the applied toxin. Conversely, G. aparine has very thin and delicate leaves, with many stiff but fragile hairs, that could more easily absorb the applied solutions. All the other leaves tested were sensitive to the encapsulated OA, with the fast and clear appearance of necrosis. The encapsulated OA was also active when applied at a concentration 1/5 lower, albeit to a lesser extent. Applied to the abaxial leaf surface, formulated OA was less active on all leaves, compared to application to the upper leaf side. In fact, when applied to $1 / 5$ concentration to the lower leaf side, the formulated OA was almost inactive. Applied alone, OA showed low activity on all the 
disks tested whereas when the OA and MSNPs were mixed together exogenously there was almost no activity.

The main objective in this assay was to ascertain if the toxicity of the nanoencapsulated toxin was really due to its "form" and not to an additive/synergic action of the two components (toxin and particles). Thus the "mixed" components (\# 4) obtained by mixing the pure toxin with the unloaded MSNPs were also tested. Moreover, droplets were applied also on the abaxial leaf surface (\# 5 and \# 6). Finally, the nanoencapsulated toxin was also tested at a 1/5 of the initial concentration. The formulated toxin proved to be highly active when applied to wounded leaf disks, causing the rapid appearance of clear and sufficiently wide necrosis to all the plants tested.

It appears quite clear that the toxicity of the ophiobolin A in the MSNPs is due to either protection of the compound, or differences in delivery, and not merely due to the presence of both components. 


\section{Discussion}

Nanoformulation for agrochemicals is the most immediate application of nanoscience in weed control one could think about. Currently, most agrochemicals are applied to crops by spraying and/or broadcasting. Usually only a very low amount of chemicals (far below the minimum effective concentration required) actually reaches the target site of crops due to problems such as leaching of chemicals, degradation by photolysis, hydrolysis and microbial degradation. Hence, repeated applications could be necessary to have an effective control which might cause unfavorable effects such as soil and water pollution.

A controlled release of the herbicide, either regulated by time, location or triggered under certain circumstances, makes this approach very attractive for future developments. Such an approach could use a variety ofl nanodevices based on different basic frames such as nanoparticles, nanocapsules, nanoclays, or liposomes (Pérez-de-Luque and Rubiales, 2009; Pérez-de-Luque and Hermosín, 2012). The active compound can be either attached to the surface, or loaded inside a nanocarrier for protection against degradation. Additionally, specific targetting ligands could be added to the nanocarrier, or those which trigger release of an active compound under certain conditions.

MSNPs have previously been studied for the storage and controlled release of the fungicide metalaxyl. The fungicide, loaded into MSNP pores from an aqueous solution by a rotary evaporation method, was released into soil and water very slowly over 30 days. In other studies, MSNPs proved to be effective carriers for the delivery of the natural pesticide avermectin, being able to protect the $\mathrm{Al}$ against UV degradation and to slow its release, dependent on the pore diameter and shell thickness. 
Figures

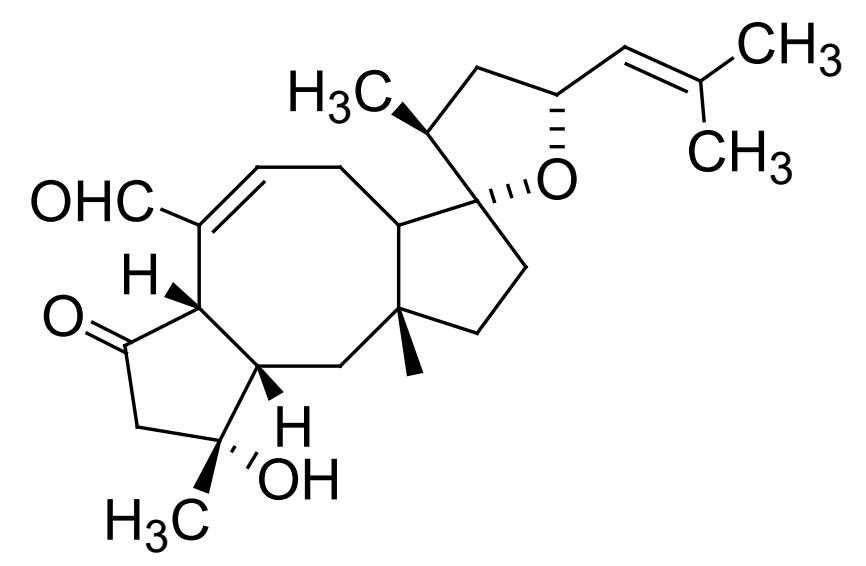

Ophiobolin A

Figure 1. Structure of Ophiobolin A 


\section{References}

Au, T.K., Wallace, S.H., Leung, P.C, 2000. The biology of ophiobolins. Life Science, 67, 733-742.

Bravo M., Preston G., Van der Hoorn R., Townley H.E., Thompson I.P., 2018. Species-specific antimicrobial activity of essential oils and enhancement by encapsulation in mesoporous silica nanoparticles. Industrial Crops and Products

Bravo M., Preston G.M., Van der Hoorn R., Flanagan N., Townley H.E., Thompson I., 2018. Enhancing cinnamon essential oil activity by nanoparticle encapsulation to control seed pathogens. Industrial Crops and Pathogens, 124: 755-764.

Bury, M., Girault, A., Megalizzi, V., Spiegel-Kreinecker, S., Mathieu, V., Berger, V., Evidente, A., Kornienko, A., Gailli, P., Vandier, P., Kiss, R., 2013. Ophiobolin A induces paraptosis-like cell death in human glioblastoma cells by decreasing BKCa channel activity Cell Death Dis., e561.

Bury, M., Novo-Uzal, E., Andolfi, A., Cimini, S., Wauthoz, N., Heffeter, P., Lallemand, B., Avolio, A., Delporte, C., Cimmino, A., Bubois, J.,. Van Antwerpen, P., Zonno, M.C., Vurro, M. Poumay, Y., Berger, W., Evidente, A., De gara, L., Kiss, R., Locato, 2013. Ophiobolin A, a sesterterpenoid fungal phytotoxin, displays higher in vitro growth-inhibitory effects in mammalian than in plant cells and displays in vivo antitumor activity. International Journal of Oncology, 43, 575-585.

Cantrell CL, Dayan FE and Duke SO, Natural products as sources for new pesticides. $J$ Nat Prod 75(6):1231-1242 (2012).

Copping LG and Duke SO, Natural products that have been used commercially as crop protection agents - a review. Pest Manag Sci 63:524-554 (2007).

Diaconu, M., Tache, A., Ana-Maria, S., Eremia, V., Gatea, F., Litescu, S., Radu, G.L., 2010. 525 Structural characterization of chitosan coated silicon nanoparticles -A FT-IR approach. U.P.B. Sci. Bull. Ser. B Chem. Mater. Sci. 72, 115-122.

Evidente, A., Andolfi, A., Cimmino, A., Vurro, M. Fracchiolla,M. Charudattan, R, 2006a. Herbicidal Potential of Ophiobolins Produced by Drechslera gigantea. J. Agric. Food Chem., 54, 17791783

Evidente, A., Andolfi, A., Cimmino, A., Vurro, M., Fracchiolla, M., Charudattan, R., Motta, A., 2006b. Ophiobolin $\mathrm{E}$ and 8-epi-ophiobolin $\mathrm{J}$ produced by Drechslera gigantea, a potential mycoherbicide of weedy grasses. Phytochemistry, 67, 2281-2287.

Huang X., Cavalcante D., Townley H., 2020. Macrophage-like THP-1 cells show effective uptake of silica nanoparticles carrying inactivated diphtheria toxoid for vaccination. Journal Nanoparticle Research, 22: 23.

Huang X., Townley H.E., 2020. An assessment of mesoporous silica nanoparticle architectures as antigen carriers. Pharmaceutics, 12: 294.

Huang X., Young N., Townley H.E., 2014. Characterization and comparison of mesoporous silica particles for optimized drug delivery. Nanomater. Nanotechnol., 4: 2.

Igor I. Slowing, Juan L. Vivero-Escoto, Chia-Wen Wu, Victor S.-Y. Lin, 2008. Mesoporous silica nanoparticles as controlled release drug delivery and gene transfection carriers. Advanced Drug Delivery Reviews, 60, 11, 1278-1288. https://doi.org/10.1016/j.addr.2008.03.012.

Kwon, S., Singh, R.K., Perez, R.A., Abou Neel, E.A., Kim, H.-W., Chrzanowski, W., 2013. Silicabased mesoporous nanoparticles for controlled drug delivery. J. Tissue Eng. 4. 561 https://doi.org/10.1177/2041731413503357

Masi, M., Dasari, R., Evidente, A., Mathieu, V., \& Kornienko, A. (2019). Chemistry and biology of ophiobolin A and its congeners. Bioorganic \& Medicinal Chemistry Letters, 29(7), 859-869.

Morrison R., Gardiner C., Evidente A., Kiss R., Townley H., 2017. Incorporation of Ophiobolin A into novel chemoembolization particles for cancer cell treatment. Pharmaceutical Research, 31(10): 2904-2917. 
Pérez-de-Luque A., Hermosín C., 2013. Nanotechnology and its use in agriculture. In: Bagchi D., Bagchi M., Moriyama H., Shahidi F. (Eds) Bio-Nanotechnology: a revolution in food, biomedical and health sciences. Wiley-Blackwell, chapter 20, p383-398.

Pérez-de-Luque A., Rubiales D., 2009. Nanotechnology for parasitic plant control. Pest Management Science 65:540-545.

Trewyn B.G., Slowing I.I., Giri S., Chen H.-T., Lin V. S.Y., 2007. Synthesis and functionalization of a mesoporous silica nanoparticle based on the sol-gel process and applications in controlled release. Acc. Chem. Res., 40, 846-853.

Vurro M., Miguel-Rojas C., Pérez-De-Luque A., 2019. Safe nanotechnologies for increasing effectiveness of environmentally friendly natural agrochemicals. Pest Management Science, 75(9): 2403-2412.

Zatout R., Masi M., Sangermano F., Vurro M., Zonno M.C., Santoro E., Calabrò, V., Superchi S., Evidente A. 2020. Drophiobiolins A and B, Bioactive Ophiobolan Sestertepenoids Produced by Dreschslera gigantea. Journal of Natural Products doi.org/10.1021/acs.jnatprod.0c00836 